\title{
Interactive comment on "Substantial ozone enhancement over the North China Plain from increased biogenic emissions due to heat waves and land cover in summer 2017' by Mingchen Ma et al.
}

\section{Mingchen Ma et al.}

yanggao@ouc.edu.cn

Received and published: 11 August 2019

This study investigates the impacts of the meteorological factors and isoprene emissions on two severe ozone pollution episodes in North China Plain (NCP) by combining ground-level observations and WRF-CMAQ simulations. The effects of hot, dry and stagnant weather conditions, as well as varied factors influencing isoprene emissions, such as land cover change, high vapor pressure deficit (VPD), and urban landscape, are examined to explain the causes of ozone pollution episodes. Results show that urbanization and land cover change made significant contributions to the enhancement 
of MDA8 ozone in the past decades. Such effects have not been considered in most of previous studies. The topic of this study well fits the scope of ACP, however, some revisions are required before the acceptance.

RE: We thank the reviewer for the constructive comments to help us further improve the manuscript. Please see the detailed responses to your comments below.

1. GENERAL The main flaw of this study is the confusion of time scales. Land cover change and urbanization usually occurs at annual to decadal time scale. However, this study focuses on ozone episodes which happen in several days. It's not scientifically reasonable to estimate the effects of land cover or urban landscape on ozone extremes. Instead, it would be helpful to examine whether land surface changes contribute to an overall enhancement of ozone concentrations during 2010s compared to 2000s. It is clear that ozone pollution level increased fast from 2014 to 2017 (Fig. 4). However, land cover or urban landscape should not change significantly within these 4 years. As a result, the main causes of more pollution episodes are related to anthropogenic emissions, atmospheric transport, and/or weather conditions, instead of land cover change.

RE: We agree that the changes of land cover and urbanization is on the scale of annual to decade. We also agree that the main causes of pollution episodes are related to anthropogenic emissions, atmospheric transport, and/or weather conditions, instead of land cover change. This is exactly what we have described in the main manuscript. For instance, in section 3.3 (Lines 366-370), we stated that "Zooming into the two ozone episodic events (June 14-21, June 26-July 3), the mean MDA8 values of case 4 are 98.02 ppbv, 108.89 ppbv, 95.75 ppbv, and 98.98 ppbv for NCP, Beijing, Hebei and Tianjin, respectively, during the heat wave periods (June 14-21, 2017; June 26-July 3, 2017), whereas the MDA8 ozone value for the case (case 1) without biogenic emission are $87.15 \mathrm{ppbv}, 93.06 \mathrm{ppbv}, 84.78 \mathrm{ppbv}$ and $89.65 \mathrm{ppbv}$ for the corresponding region." This indicates that the contributions, i.e., anthropogenic emissions, transport, etc, except biogenic emissions accounted for the majority (more than $80 \%$ ) of ozone

Interactive

comment
Printer-friendly version

Discussion paper

2 
formation. Although the absolute contribution of biogenic emissions is smaller than the anthropogenic emissions, the substantial ozone increment is important in both understanding the mechanisms of high ozone events and ozone pollution control. To make this clear, we added a statement in the revised manuscript, "the underestimation of biogenic emission due to changes in land cover may be exaggerated in years with high temperatures and high VPD" (Lines 290). To further address the reviewer's concern regarding the effect of land cover over the recent years, we conducted another sets of simulations (the same as cases 2-4 discussed above) during June 8 th to mid-July in 2016, similar period as 2017. The mean MDA8 ozone concentrations over NCP during this entire period in 2017 for case 2 is 79.03 ppbv, and statistical significant enhancement (1.34 ppbv) was achieved in case 3 . In comparison to case 3 , the land cover change in case 4 shows statistical significant increase as well (1.13 ppbv). Similarly, looking at the entire period in 2016 (June 8th - July 4th), statistical significant, and even higher in relative to 2016, increase was achieved in case 3 ( 1.55 ppbv) compared to case 2 (90.11 ppbv), and case 4 (1.23 ppbv) compared to case 3 . We have added the discussions from Line 405-414 in the revised manuscript.

2. In addition, most of the isoprene observations used by this study were obtained more than ten years ago, making it difficult to evaluate the impacts of land cover change and urban landscape on BVOC emissions, which are the major merits of this study. From this aspect, these changes made limited contributions to the high ozone episodes in 2017 , especially compared to the year 2014 (NOT 2003). And the title of the paper is inaccurate.

RE: We thank the reviewer for putting forward the concern of isoprene observations. In fact, we realized the importance of isoprene evaluation as well as the data limitation during our study period. Therefore, we have tried our best to search the isoprene observations from the previous literature. As far as we know, we have added the observational data available to us. Indeed, we did used the most recent observations (Fig. 7B) in 2016 and 2017. It clearly displays the cases without urban landscape 
substantially underestimated the isoprene emissions. We believe the evidence is clear and also agree that more isoprene observations and evaluations are needed in future. As we addressed in the previous response, we emphasize the underestimation of biogenic emission due to changes in land cover may be exaggerated in years with high temperatures and high VPD. From the point of view, we consider the title is fine.

3. Page 3, line 69. The North China Plain should be defined with specific latitudes and longitudes or detailed descriptions.

RE: The defined latitudes and longitudes of the North China Plain have been added.

4. Page 8, line 194. The author could consider remove the word "medium" because the medium ozone pollution events are not analyzed.

$\mathrm{RE}$ : This has been corrected in the revised manuscript.

5. Page8, line 200. Fig. 1 is in the text rather than supporting information.

$\mathrm{RE}$ : "In the supporting information" has been deleted.

6. Page 8, lines 219-220. The sentence "Please note that..." is unnecessary and should be removed.

RE: This sentence has been deleted in the revised manuscript.

7. Page 11, Fig.4. The coordinate and scale for the daily total precipitation (yellow bars) are not shown.

$\mathrm{RE}$ : The coordinate of the daily precipitation is the same as Tmax in the right side, and it is marked in the coordinate title (yellow).

8. Page 11. Fig. 4. It would be better that the time series of VPD are exhibited, because the article discusses the effect of VPD on isoprene emissions in Sect.3.3.

RE: Thanks for the suggestion. We have added the VPD time series in Fig. S4 in the supporting information. 
9. Figure 4: In addition to the two episodes examined in 2017, the similar hot, dry and stagnant weather condition shown several times during 2014-2017, such as 16th - 22th July in 2014, 6th-14th July in 2015 and 8th-16th July in 2017. Why these favorable conditions do not result in ozone episodes?

RE: We did notice the other episodes with relatively similar meteorological conditions, however, a few differences exist. For instance, albeit of the high temperature during July 16-22 2014, there was continuous rainfall over NCP, weakening the solar radiation and subsequent ozone formation. During July 6-14 2015, there is a relatively short period with high temperature (i.e., July 11 to 13), however, rainfall occurs from July 14 , breaking the heat event. Although temperature during July 8th to 20th is relatively high, wind velocity during July 8th and 9th is too high to favor the ozone accumulation. In the following two days, the slow wind with high surface temperature provide appropriate photochemical reaction conditions which are conductive to ozone accumulation. The increased precipitation with reduced solar radiation from July 14th make the high ozone events unlikely occur.

10. Page 15, Fig.7A. The difference between case 4 and case 5 is $15 \%$ increase in isoprene emissions in Beijing, but why the simulated isoprene concentration is much higher in case 5 compared with it in case4?

$\mathrm{RE}$ : Please be aware that the observations of isoprene concentrations are located in the urban area of Beijing. Although the absolute magnitude of urban isoprene emission is not too large (accounting for 15\%), the location of the emissions plays a much larger role. As we know, most of the isoprene emissions from the forest in Beijing is located in the rural area, which is relatively far from the urban area. Considering the short lifetime of isoprene (about 2 hours), it may not be very efficient for these isoprene emissions to transport to the urban areas. Instead, the isoprene directly emitted from the urban areas may contribute to the isoprene concentration very efficiently. Therefore, the $15 \%$ increase in isoprene emission contributes to the substantial increase of urban isoprene concentration. 
11. Page 16, Fig.8. The abbreviations of NMB, NME, MFB and MFE should be explained in the figure capture. The same issue could be considered in text line 343.

RE: These abbreviations have been added in the revised manuscript.

Interactive comment on Atmos. Chem. Phys. Discuss., https://doi.org/10.5194/acp-2019-362, 2019. 